\title{
Electromyography and asymmetry index of masticatory muscles in undergraduate students with temporomandibular disorders
}

\author{
Gisele Harumi Hotta ${ }^{1}$, Ana Izabela Sobral de Oliveira ${ }^{1}$, \\ Anamaria Siriani de Oliveira ${ }^{1}$, Cristiane Rodrigues Pedroni ${ }^{2}$
}

${ }^{1}$ Universidade de São Paulo - USP, Ribeirão Preto Medical School, Department of Biomechanics, Medicine and Rehabilitation of the Locomotor Apparatus, Ribeirão Preto, SP, Brazil

2Universidade Estadual Paulista - UNESP, School of Philosophy and Sciences, Department of Physical Terapy and Occupational Therapy, Marilia, SP, Brazil

\begin{abstract}
Aim: To compare the electromyographic activity and the asymmetry index among degrees of severity of temporomandibular disorders (TMD). Methods: Surface electromyography (EMG) of the right and left masseter and temporalis muscles was performed in 126 undergraduate students at rest and at maximal voluntary contraction. Three measurements were performed for five seconds of maximal contraction and mandibular rest. The degree of temporomandibular dysfunction was identified according to the Fonseca anamnestic index. The analysis of the asymmetry index for two pairs of muscles during maximal voluntary contraction was based on the asymmetry index proposed by Naeije, McCarrol and Weijs (1989). Results: $48.41 \%$ of the sample had mild TMD, followed by volunteers without TMD (26.98\%), moderate TMD (19.05\%) and severe (5.56\%). The survey results show absence of correlation between the Fonseca anamnestic index and electromyographic activity at rest and at maximal voluntary contraction in undergraduate students in both muscles $(p>0.05)$ and the asymmetry index did not differ between the analyzed groups. Conclusions: For the population of undergraduate students, there is no evidence that the presence and severity of TMD influence the EMG activity of masseter and temporalis muscles and the muscle asymmetry index at rest and maximal voluntary contraction.
\end{abstract}

Keywords: electromyography; evaluation; stomatognathic system.

\section{Introduction}

Temporomandibular dysfunction (TMD) is a disorder of the stomatognathic system characterized by the presence of pain in the preauricular region, fatigue of masticatory muscles (MM), limitation or deviation during movement of the temporomandibular joint (TMJ) and could be associated to noise during opening and closing the mouth ${ }^{1-2}$. The difficulty of identifying the TMD is related to its multifactorial etiology and the complex relationship of TMJ with other structures of the head, neck and scapular waist ${ }^{3}$, apart from an important relationship with emotional factors, especially in adolescents ${ }^{2,4}$. The main muscles affected in this dysfunction are the temporal (anterior and posterior), masseter (superficial and deep), pterygoid (medial and lateral) and digastric muscles ${ }^{5}$.

The prevalence of TMD symptoms has been shown to vary from 16 to 59\% in general populations ${ }^{1}$ while other studies showed that nearly $70 \%$ of 
undergraduate students present some symptoms of $\mathrm{TMD}^{3,6}$. Despite such a high prevalence, only $7 \%$ of subjects classified as moderate and severe TMD patients reported to had ever sought medical care ${ }^{6}$. Undergraduate students are vulnerable to signs and symptoms of $\mathrm{TMD}^{6}$, but the future impact of these symptoms on this population is unknown.

Several questionnaires were prepared for the assessment of TMD signs and symptoms, and currently the most widely used method for classification is the Research Diagnostic Criteria for Temporomandibular Disorders (RDC/TMD) $)^{4}$. Nevertheless, the index proposed by Helkimo ${ }^{7}$ (1994) has been clinically used to classify the severity levels of TMD, especially in epidemiological survey studies ${ }^{1-4,6}$. Fonseca et al. ${ }^{8}$ (1994) based on the Helkimo index ${ }^{7}$ translated and validated the questionnaire into Portuguese with $95 \%$ reliability and good correlation with the Helkimo index. The Fonseca et al. ${ }^{8}$ (1994) questionnaire has the advantage of being self-manageable, short application time, low cost and may be used for the screening of patients ${ }^{1-2,6}$.

Surface electromyography is widely used for evaluation and observation of the electrophysiological behavior of the muscles under different physiological conditions ${ }^{4-5}$. This method is a non-invasive evaluation of the bioelectric phenomena of muscle activity at rest and during muscle contraction and is useful for assessing the electrical activity of $\mathrm{MM}^{9-10}$. Research of electromyographic characteristics among undergraduate students with TMD is essential for an early detection of bioelectric characteristics of muscles that may predispose individuals to pain and muscle dysfunction and/or joint in the adult age $\mathrm{e}^{4,9}$.

Electromyography studies in MM have shown that the mean values for electrical activity of the masseter and temporal muscles at rest range $2.2-4.0 \mathrm{~V}$ in individuals with TMD and 2.9 3.8 V in healthy subjects, without a significant difference among the groups ${ }^{10-11}$. However, Glaros et al. ${ }^{12}$ (1997) state that the electrical activity is slightly higher in the TMD patients, averaging 2.9 to $3.3 \mathrm{~V}$ for the masseter and 4.5 to $5.7 \mathrm{~V}$ for temporal. In healthy volunteers, the average was 2.2 to 2.3 for the masseter and 3.6 to 3.7 for the temporal. In literature, the consensus is that the temporal muscle has a higher electrical activity at rest compared with the masseter ${ }^{10-12}$. At maximal voluntary contraction (MVC), adult TMD patients have a lower electrical activity, ranging from 64.1 to $79.2 \mathrm{~V}$, compared to healthy subjects, ranging from 140.2 to $182.8 \mathrm{~V}^{10-11}$, but such values have not been found for the college age group.

In this context, the aim of this study was to compare the electromyographic activity and the asymmetry index with the severity degrees of TMD.

\section{Material and methods}

\section{Participants}

The convenience sample was recruited from the São Paulo State University - UNESP, Marilia, Brazil. Individuals using orthodontic appliances or orthopedic functional, fixed or removable dentures were excluded from the study and also abusers of painkiller-type drugs, anti-inflammatory drugs, muscle relaxants and antidepressants, as well as those with some type of neurological disorder.

This study was approved by the Ethics in Research Committee of the São Paulo State University in accordance with Resolution of the National Health Council 196/96 (reference number: 0457/2012) and the rights of the subjects were protected. All participants were informed about the study and signed an informed consent form before participating.

\section{Instruments and assessment procedures}

\section{Fonseca Anamnestic Index (IAF)}

The IAF was initially applied to each individual by a physical therapist blinded to the electromyographic evaluation, determining the presence and severity of TMD. The questionnaire consists of 10 questions, where the YES answer scores 10, SOMETIMES scores 5 and NO scores 0 . The sum of the answer values corresponds to the following classification: 0-15 (no TMD), 20-40 (mild TMD), 45-65 (moderate TMD) and 70-100 (severe TMD).

\section{Surface Electromyography}

Simultaneous sampling of signals was used to record the electromyographic signals of the MM (bilateral masseter and anterior temporal muscles) by the Lynx Technology LTD (Lynx ${ }^{\circledR}$ São Paulo, SP, Brazil) A/D board, composed of six active channels with analog band pass filters with cut off frequency at 20-1000 $\mathrm{Hz}$ and digitized with 16-bit resolution. The equipment was connected to a battery with a capacity of $10 \mathrm{Ah}, 12 \mathrm{~V}$, to reduce the noise from the power supply. The guidelines of Surface Electromyography for Non-Invasive Assessment of Muscles (SENIAM) were followed to prepare the $\operatorname{skin}^{13}$

During signal acquisition, the subjects remained comfortably seated in a chair with back support and no support for the head and upper limbs, maintaining the hands rested on the femur, the soles of the feet flat on the floor with the knees in a $90^{\circ}$ flexion, head erect and looking to the horizon. The volunteer got no visual feedback from the computer screen. Before each data collection, the volunteer underwent familiarization with the equipment and the movements to be performed, getting all the necessary instructions and information.

The electrodes were placed bilaterally along the anterior fibers of the temporal and masseter muscles ${ }^{14}$. The recordings were made in the resting mandibular position and maximal voluntary contraction (MVC) with the use of Parafilm $M{ }^{\circledR}$ (Wertheim, Baden-Württemberg, Germany) folded similarly to a Trident gum $(3.5 \times 1.5)^{15}$ and placed bilaterally between the premolars, first and second mandibular and maxillary molars. There were three measurements during MVC. All recordings were performed for $5 \mathrm{~s}$ with a two-minute interval between them.

The data acquisition and storage of digitized signals were performed by Aqdados software $\left(\operatorname{Lynx}{ }^{\circledR}\right)$, version 7.2 
for Windows. The used sensors were passive surface double halves of silver/silver chloride circles, adhesive, disposable, $42 \mathrm{~mm}$ long, $20 \mathrm{~mm}$ wide, $20 \mathrm{~mm}$ from center to center (Miotec ${ }^{\circledR}$ Porto Alegre, RS, Brazil), coupled to a Lynx Technology LTD preamp with a $10 \mathrm{G} \Omega$ input impedance, $130 \mathrm{~dB}$ common rate of rejection and gain of 20 times.

\section{Data Analysis}

The electromyographic signal was processed in the time domain by root mean square (RMS). All registration procedures and analyses of the electromyographic signal followed the Standards for Reporting EMG Data ${ }^{16}$. Data from the MVC and at rest were standardized on the average obtained by three repetitions of the MVC.

The statistical program used was SPSS Statistics Version 17.0 for Windows (SPSS Inc, Chicago, IL). The KolmogorovSmirnov test was used to verify the normality of data. The Kruskal-Wallis test was used for comparison between groups. The Spearman correlation coefficient was used to calculate the association between variables. For all data processing the significance level was set at $5 \%$.

The asymmetry values of muscle activity of the two muscle pairs during isometric contraction were based on the asymmetry index (AI), proposed by Naeije, McCarrol and Weijs $^{17}$ (1989). If the muscle activation level is fully symmetrical IA is $0 \%$, while the full asymmetry corresponds to $100 \%$. The formula is: IA $=[(\mathrm{RMS}$ right - left RMS $) /$ (RMS right + left RMS) $* 100]$.

\section{Results}

The clinical and demographic characteristics of the sample are shown in Table 1 . The participants were 126 undergraduate students with mean age of $21.59( \pm 5.21)$ years and $74.24 \%$ of the sample were females. Among the students, $34(26.98 \%)$ presented no TMD, $61(48.41 \%)$ mild TMD, 24 $(19.05 \%)$ moderate TMD and $7(5.56 \%)$ severe TMD.

Comparing the groups according to the IAF severity and disregarding the right and left sides, the RMS values of electromyography activity showed no difference $(\mathrm{p}<0.05)$ for the masseter and temporalis at rest (Figures 1 and 3 ) and in MVC (Figures 2 and 4).

The analysis of the electromyographic activity and the IAF carried out by Spearman correlation coefficient showed that there is no association between the variables. Correlation was not found between the RMS value of the masseter and IAF $(\mathrm{r}=-0.066)$, and the temporal and IAF with MVC $(r=-0.081)$.

Table 1: Sample characterization according to the score obtained from the Fonseca anamnestic index ${ }^{8}$

\begin{tabular}{llll}
\hline Groups & Sample Size & \multicolumn{1}{c}{ Age } & \multicolumn{1}{c}{ IAF } \\
Without TMD & $34(26.98 \%)$ & $21.59 \pm 5.21$ & $9.56 \pm 4.33$ \\
Mild TMD & $61(48.41 \%)$ & $21.15 \pm 2.85$ & $27.79 \pm 6.31$ \\
Moderate TMD & $24(19.05 \%)$ & $21.69 \pm 3.40$ & $55 \pm 6.35$ \\
Severe TMD & $7(5.56 \%)$ & $22 \pm 2.55$ & $80.63 \pm 9.43$ \\
\hline
\end{tabular}

TMD: Temporomandibular dysfunction; IAF: Fonseca anamnestic index.

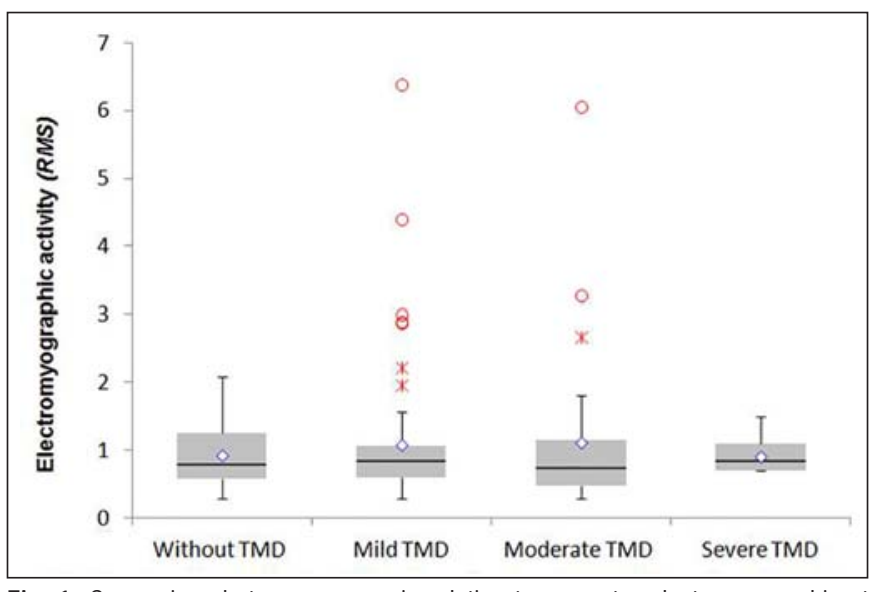

Fig. 1: Comparison between groups in relation to masseter electromyographic at rest. TMD: temporomandibular dysfunction

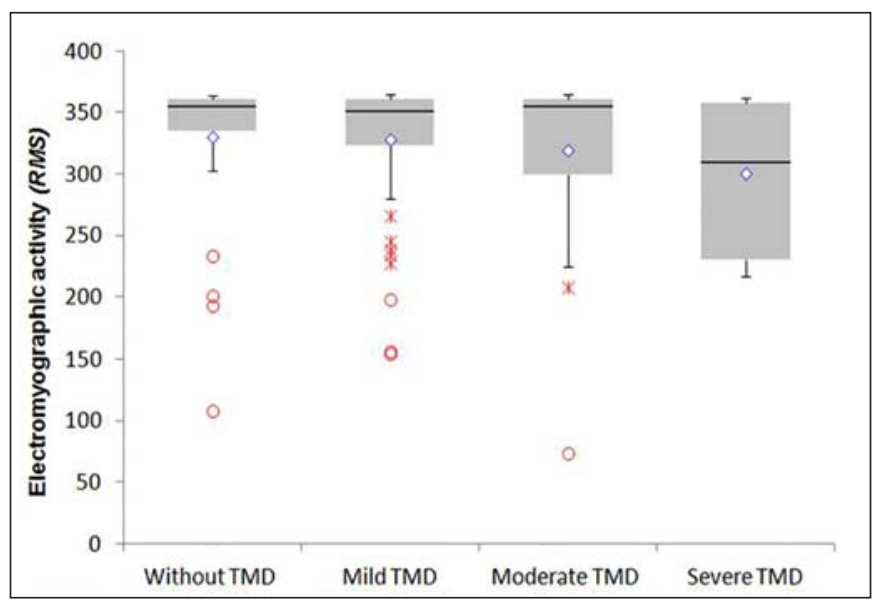

Fig. 2: Comparison between groups in relation to masseter electromyographic activity at maximal voluntary clench. TMD: temporomandibular dysfunction

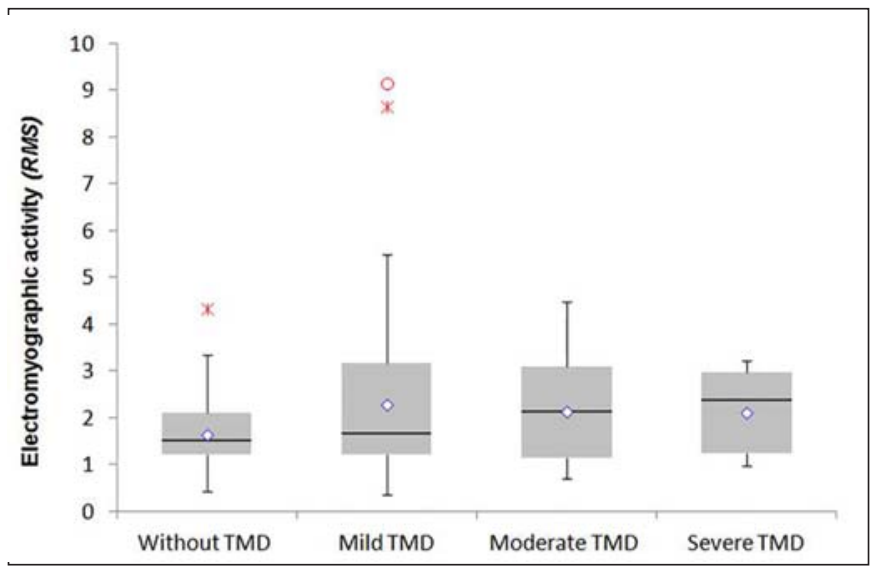

Fig. 3: Comparison between groups in relation to temporal electromyographic activity at rest. TMD: temporomandibular dysfunction

There is no evidence that the temporal $(\mathrm{r}=0.063)$ and the masseter $(r=-0.001)$ correlate with the IAF at rest (Table 2).

The groups showed a degree of asymmetry index with greater evidence at rest, however, statistical differences were not found between groups. Descriptive data are presented in Table 3. 


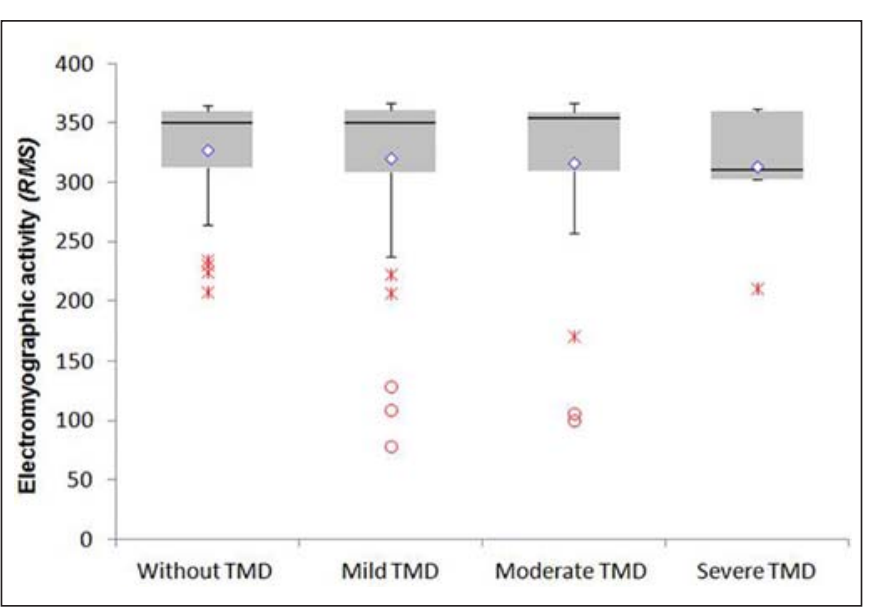

Fig. 4: Comparison between groups in relation to temporal electromyographic activity at maximal voluntary clench. TMD: temporomandibular dysfunction

Table 2: Correlation between the Fonseca anamnestic index and electromyographic activity at rest and at maximal voluntary contraction.

\begin{tabular}{lll}
\hline Correlation & $\boldsymbol{r}_{\boldsymbol{s}}$ & $\boldsymbol{p}$ valor \\
IAF (score) X MEAR $(\mathrm{rms})$ & -0.001 & 0.99 \\
IAF (score) X TEAR $(\mathrm{rms})$ & 0.063 & 0.47 \\
IAF (score) X MEAMVC $(r m s)$ & -0.066 & 0.44 \\
IAF (score) X MEAMVC $(r m s)$ & -0.081 & 0.35
\end{tabular}

IAF: Fonseca anamnestic index; MEAR: Masseter's electromyographic activity at rest; TEAR: Temporal electromyographic activity at rest; MEAMVC: Masseter electromyographic activity at maximal voluntary contraction; TEAMVC: Temporal electromyographic activity at maximal voluntary contraction. $r_{s}$. Spearman's correlation coefficient

\section{Discussion}

The study results showed that among the undergraduate students population, there are many individuals with some type of TMD signs or symptoms. The EMG activity of the masseter and temporal does not differ between the presence or absence of symptoms and between different degrees of TMD, both at rest and the maximal voluntary contraction (MVC). There is also no evidence of changes in muscle asymmetry index at rest and MVC in individuals with signs and symptoms of TMD. There is no correlation between the score obtained by IAF and the EMG activity of masseter and temporalis muscles.

The high incidence of mild TDM in the study population corroborates those of studies that classify mild TMD as the most prevalent type among Brazilian male and female undergraduate students ${ }^{1-2}$. This large number of undergraduate students with TMD signs or symptoms emphasizes the importance of studying TMD in this age group, considering possible progression of the disorder and future need of specialized medical care ${ }^{2,6}$.

The results of this study are consistent with those found of Hugger et al. ${ }^{10}$ (2012) and Weggen et al. ${ }^{18}$ (2012), who analyzed EMG activity in MVC and did not identify differences between groups of healthy participants and those with $\mathrm{TMD}^{10,18}$. This finding is justified by the fact that to create accurate jaw movements, entries of various sensory receptors must be received by the central nervous system by afferent nerve fibers. The brain assimilates and organizes this information and generates appropriate motor activities by the efferent nerve fibers. These motor activities involve the contraction of some muscle groups and the inhibition of others. Chewing is a subconscious activity, but it may be controlled consciously at any moment ${ }^{5}$ declaring the individual's ability to control the use of the $\mathrm{MM}$ in unnecessary activities, avoiding increase in electrical activity of the muscle in this phase.

Healthy subjects when compared with individuals with TMD exhibit reduction of electrical potentials of the masseter and temporal muscles at rest ${ }^{19-21}$. However, these differences are not consistent in the literature, and the increase of electrical potentials at rest is considered in most cases as the result of chronic pain ${ }^{10}$.

Although some authors ${ }^{11,19-21}$ confirm the increased EMG activity at rest and decrease in activation capacity during MVC and it is known that during mastication the relationship between muscle actions is generally similar among individuals of the same gender. However, there are significant differences in bite between men and women and between young and adults, ${ }^{5}$ justifying the difference between the results of this study with the above mentioned, since the populations consisted of different age groups.

The chewing movements of patients with myofascial pain had the same pattern of healthy individuals. Thus, people with pain in $\mathrm{MM}$ or joint noises may have normal mandibular range of motions.

In both groups at the start of the closing phase of mastication, the ipsilateral side of the temporal muscle to the bite is first activated and then the contralateral temporal muscle and masseter muscles act simultaneously. The EMG activity in these muscles is very low, but it increases gradually,

Table 3: Descriptive data of the asymmetry index, according Naije et al ${ }^{17}$ (1989).

\begin{tabular}{lllll}
\hline & \multicolumn{2}{c}{ Masseter } & \multicolumn{2}{c}{ Temporalis } \\
& Resting (\%) & MVC (\%) & Resting (\%) & MVC (\%) \\
Without TMD & $17.29 \pm 18.10$ & $3.47 \pm 7.32$ & $22.61 \pm 16.83$ & $5.85 \pm 9.15$ \\
Mild TMD & $14.65 \pm 15.81$ & $5.28 \pm 9.75$ & $16.93 \pm 16.61$ & $5.16 \pm 8.97$ \\
Moderate TMD & $19.24 \pm 25.05$ & $8.53 \pm 16.23$ & $27.82 \pm 19.42$ & $5.00 \pm 8.46$ \\
Severe TMD & $20.51 \pm 23.71$ & $12.83 \pm 13.61$ & $29.36 \pm 26.96$ & $7.27 \pm 11,93$ \\
\hline
\end{tabular}

Data expressed as mean \pm standard deviation. MVC: Maximal voluntary contraction; TMD: Temporomandibular dysfunction. 
reaching a peak at the end of the closing movement ${ }^{5}$.

The muscle activity changes during MVC and at rest are relatively small, but these small changes in muscle activity at rest were reported as clinical indicators of TMD, but considering these small changes in EMG activity as a factor in the pathogenesis of muscle pain in these patients is still controversial $^{22}$.

The muscle tension is characterized as causal factor for TMD, therefore the electrical activity is not necessarily increased in the MM on these patients. However, it should be stressed that a possible increase in muscle activity may precede and produce pain, and that once a group of motor units are tired or painful they are centrally inhibited from being recruited ${ }^{22}$.

The electromyographic data from this study, along with the data from Carlson et al. ${ }^{22}$ (1998) do not support the use of EMG on an integrated surface as a means to differentiate patients with TMD from controls, even when they are exposed to standard stresses with a significant level of physiological and emotional activation ${ }^{23}$.

In most studies the EMG activity is increased at rest for TMD patients, corroborating the above information. Nevertheless, Chandu et al. ${ }^{23}$ (2004) and Hugger et al. ${ }^{10}$ (2012) concluded that the isolated assessment of EMG activity of the masseter and temporal muscles is not able to evaluate and distinguish the TMD patients from orofacial pain-free individuals.

The muscle asymmetry during chewing activity is seen predominantly in patients with $\mathrm{TMD}^{24}$. However, RodriguesBigaton et al. ${ }^{25}$ (2010) found no significant difference between TMD or healthy patients when assessing muscle asymmetry. It is observed that all individuals including the healthy ones have some degree of MM asymmetry, and that the temporal muscle is more active compared to the masseter in all subjects ${ }^{1024-25}$.

Tartaglia et al. ${ }^{21}$ (2011) found that the EMG activity of the masseter, temporalis and sternocleidomastoid muscles is less symmetrical in TMD patients, however this difference is not significant, while Hugger et al. ${ }^{10}$ (2012) report that there is no difference between the groups as regards symmetry of the masseter muscle activity, but analyzing the temporal muscle this difference is verified.

There is no difference between TMD patients and healthy individuals when observing asymmetry at rest and it can be seen that healthy individuals exhibit a significant dominance of temporalis in relation to the masseter muscles. However, this prevalence does not exist while analyzing asymmetry during CVM, additionally, dominance between the sides in the same patient was found in both groups ${ }^{25}$.

In conclusion, for this population of undergraduate students, there is no evidence that the presence and severity of TMD influence the EMG activity of masseter and temporalis muscles and the muscle asymmetry index at rest and maximal voluntary contraction.

\section{Acknowledgements}

The authors are grateful to São Paulo State University and Pró-Reitoria de Pesquisa (PROPE) for financial support. No conflicts of interest declared.

\section{References}

1. Bevilaqua-Grossi D, Chaves TC, de Oliveira AS, Monteiro-Pedro V. Anamnestic index severity and signs and symptoms of TMD. Cranio J Craniomandib Pract. 2006; 24: 112-8.

2. De Oliveira AS, Dias EM, Contato RG, Berzin F. Prevalence study of signs and symptoms of temporomandibular disorder in Brazilian college students. Braz Oral Res. 2006; 20: 3-7.

3. Pedroni CR, De Oliveira AS, Guaratini MI. Prevalence study of signs and symptoms of temporomandibular disorders in university students. J Oral Rehabil. 2003; 30: 283-9.

4. Lauriti L, Silva PF da C, Politti F, Biasotto-Gonzalez DA, Fernandes KPS, Mesquita-Ferrari RA, et al. Pattern of electromyographic activity in mastication muscles of adolescents with temporomandibular disorder. J Phys Ther Sci. 2013; 25: 1303-7.

5. Soboïeva U, Lauriòa L, Slaidiòa A. The masticatory system-an overview. Stomatol Issued Public Inst Odontol Stud Al. 2005; 7: 77-80.

6. Oliveira AS de, Bevilaqua-Grossi D, Dias EM. Signs and symptoms of temporomandibular disorders across Brazilian regions. Fisioter Pesq. 2008 [cited 2015 May 4]; 15: 392-6. Available from: http://www.scielo.br/ scielo.php?script=sci_abstract\&pid=S1809-29502008000400013 \&lng=en\&nrm=iso\&tlng=pt

7. Helkimo M. Studies on function and dysfunction of the masticatory system. II. Index for anamnestic and clinical dysfunction and occlusal state. Swed Dent J. 1974; 67): 101-21.

8. Fonseca DM da, Bonfante G, Valle AL do, Freitas SFT de. Diagnosis of the craniomandibular disfunction through anamnesis. RGO. 1994 [cited 2015 May 4]; 42: 23-8. Available from: http://bases.bireme.br/cgi-bin/wxislind.exe/ iah/online/?IsisScript=iah/iah.xis\&src=google \& base=BBO\&lang= p\&nextAction=Ink\&expr Search=10120\&index Search=ID

9. Celinski Al, Cunali RS, Bonotto D, Farias AC de, Cunali PA. Surface electromyography for temporomandibular disorders: systematic review. Rev Dor. 2013 [cited 2015 May 4]; 14: 147-50. Available from: http: // www.scielo.br/scielo.php?script=sci abstract\&pid=S1806$00132013000200015 \&$ lng=en\&nrm=iso\&tlng=en

10. Hugger S, Schindler HJ, Kordass B, Hugger A. Clinical relevance of surface EMG of the masticatory muscles. (Part 1): Resting activity, maximal and submaximal voluntary contraction, symmetry of EMG activity. Int J Comput Dent. 2012; 15: 297-314.

11. Manfredini D, Cocilovo F, Favero L, Ferronato G, Tonello S, GuardaNardini L. Surface electromyography of jaw muscles and kinesiographic recordings: diagnostic accuracy for myofascial pain. J Oral Rehabil. 2011; 38: 791-9.

12. Glaros AG, Glass EG, Brockman D. Electromyographic data from TMD patients with myofascial pain and from matched control subjects: evidence for statistical, not clinical, significance. J Orofac Pain. 1997; 11: 125-9.

13. Hermens HJ, Freriks B, Disselhorst-Klug C, Rau G. Development of recommendations for SEMG sensors and sensor placement procedures. J Electromyogr Kinesiol. 2000; 10: 361-74.

14. De Felício CM, Ferreira CLP, Medeiros APM, Rodrigues Da Silva MAM, Tartaglia GM, Sforza C. Electromyographic indices, orofacial myofunctional status and temporomandibular disorders severity: A correlation study. J Electromyogr Kinesiol. 2012; 22: 266-72.

15. Ap Biasotto-Gonzalez D, Berzin F, da Costa JM, de Gonzalez TO. Electromyographic study of stomatognathic system muscles during chewing of different materials. Electromyogr Clin Neurophysiol. 2010; 50: 121-7.

16. Merletti R. Standards for Reporting EMG Data. J Electromyogr Kinesiol. 1999 [cited 2015 May 4]; 24: III-IV. Available from: http: // www.jelectromyographykinesiology.com/article/S1050641114000066/ abstract

17. Naeije M, McCarroll RS, Weijs WA. Electromyographic activity of the human masticatory muscles during submaximal clenching in the intercuspal position. J Oral Rehabil. 1989; 16: 63-70. 
18. Weggen T, Schindler HJ, Kordass B, Hugger A. Clinical and electromyographic follow-up of myofascial pain patients treated with two types of oral splint: a randomized controlled pilot study. Int J Comput Dent. 2013; 16: 209-24.

19. Ferrario VF, Sforza C, Colombo A, Ciusa V. An electromyographic investigation of masticatory muscles symmetry in normo-occlusion subjects. J Oral Rehabil. 2000; 27: 33-40.

20. Santana-Mora U, López-Ratón M, Mora MJ, Cadarso-Suárez C, LópezCedrún J, Santana-Penín U. Surface raw electromyography has a moderate discriminatory capacity for differentiating between healthy individuals and those with TMD: a diagnostic study. J Electromyogr Kinesiol 2014; 24: 332-40.

21. Tartaglia GM, Lodetti G, Paiva G, De Felicio CM, Sforza C. Surface electromyographic assessment of patients with long lasting temporomandibular joint disorder pain. J Electromyogr Kinesiol. 2011; 21: 659-64.

22. Carlson CR, Reid KI, Curran SL, Studts J, Okeson JP, Falace D, et al. Psychological and physiological parameters of masticatory muscle pain. Pain. 1998; 76: 297-307.

23. Chandu A, Suvinen TI, Reade PC, Borromeo GL. The effect of an interocclusal appliance on bite force and masseter electromyography in asymptomatic subjects and patients with temporomandibular pain and dysfunction. J Oral Rehabil. 2004; 31: 530-7.

24. Scopel V, Alves da Costa GS, Urias D. An electromyographic study of masseter and anterior temporalis muscles in extra-articular myogenous TMJ pain patients compared to an asymptomatic and normal population. Cranio J Craniomandib Pract. 2005; 23: 194-203.

25. Rodrigues-Bigaton D, Berni KCS, Almeida AFN, Silva MT. Activity and asymmetry index of masticatory muscles in women with and without dysfunction temporomandibular. Electromyogr Clin Neurophysiol. 2010; 50: $333-8$. 